\title{
Interstitial brachytherapy for orbital soft tissue sarcoma: an innovative technique
}

\author{
Siddhartha Laskar, MD!, Avinash Pilar, MD!, Nehal Khanna, MD!, Yogesh Ghadi, MSc² \\ 'Department of Radiation Oncology, ${ }^{2}$ Department of Medical Physics, Tata Memorial Hospital, Mumbai, India
}

\begin{abstract}
Purpose: To report an innovative technique of interstitial brachytherapy developed for treatment of orbital soft tissue tumors.

Material and methods: A 4-month-old child diagnosed with rhabdomyosarcoma of orbit was treated with multiagent chemotherapy (CTh) and brachytherapy. Pre-planning computed tomography (CT) images were obtained and clinical target volume (CTV) was defined using the pre-treatment magnetic resonance imaging (MRI). Brachytherapy plan was generated for deciding optimal catheter placement. With the child under general anesthesia, catheter entry points were extrapolated and marked on the skin as determined from the pre-planning CT scan. Implantation of catheters was performed as per pre-determined catheter position and depths. Brachytherapy plan was generated and evaluated using dose volume histograms (DVH). A comparative external beam radiotherapy (EBRT) plan using RapidArc was also generated for the CTV with a $3 \mathrm{~mm}$ margin as the planning target volume (PTV).

Results: The mean CTV dose with brachytherapy was 158\% compared to 101\% with RapidArc. The CTV V 100 was $90 \%$ for brachytherapy vs. 95\% for RapidArc. The mean dose to Lt Lens were $51 \%$ and $60 \%$, respectively for brachytherapy and RapidArc, while the corresponding mean doses to the bony orbit were $39 \%$ and $68 \%$, respectively. Follow-up MRI at 3 months showed complete response of the tumor.

Conclusions: Interstitial brachytherapy for orbit using this innovative technique is a safe and effective modality of local treatment for appropriately selected orbital soft tissue tumors. Brachytherapy resulted in excellent disease control with significant reduction of dose to surrounding ocular structures compared to EBRT.

J Contemp Brachytherapy 2017; 9, 5: 466-471 DOI: https://doi.org/10.5114/jcb.2017.70957
\end{abstract}

Key words: brachytherapy, ocular, orbit, soft tissue sarcoma.

\section{Purpose}

Orbital rhabdomyosarcoma (RMS) is the most common primary orbital malignancy in children and typically occurs in the first decade of life [1]. Till the late 70's, orbital RMS was typically managed with exenteration and was associated with poor prognosis and functional outcome. Currently, it is best managed with a combination of multi-agent chemotherapy (CTh) and external beam radiotherapy (EBRT) resulting in a 5-year survival exceeding $90 \%$ [2,3]. However, EBRT is feared (necessarily or not) by most for its late toxicities of cranio-facial deformities, visual/orbital adverse effects and neuroendocrine sequelae especially in very young children $[4,5,6]$. Attempts to delay or omit EBRT have been sought with higher rates of local recurrence [7]. Brachytherapy with its steep dose fall off, provides an advantage over EBRT in reducing doses to orbit and neurological structures, and thereby potentially reducing the late sequelae of EBRT. However, it can be technically challenging and most reports of orbital brachytherapy are in the peri-operative setting, performed after surgical debulking $[4,8,9,10,11,12,13]$. Such procedures are challenging and carry the potential morbidities of both treatment modalities, i.e. surgery and brachytherapy.

We report the technical details of an innovative technique developed at our institute for interstitial brachytherapy of the orbit.

\section{Material and methods \\ Case details}

A 4 month old male patient presented to the Tata Memorial Hospital with complaints of redness and swelling at medial canthus of the left eye, which extended along the upper and lower eyelids (Figure 1A). Magnetic resonance imaging (MRI) of the orbit showed a homogenous lesion of $2.2 \times 2.0 \times 2.2 \mathrm{~cm}$ in the extraconal compartment of medial aspect of left orbit. The lesion was limited to the orbit without any intracranial extension, or extension into the ethmoid air sinuses (Figures 1B and 1C). Biopsy was suggestive of alveolar rhabdomyosarcoma, which was positive for
Address for correspondence: Prof. Siddhartha Laskar, MD, Department of Radiation Oncology, Tata Memorial Hospital, Dr. Ernest Borges Marg, Parel, Mumbai, India - 400012, India, phone: + 9122 24177167, fax: + 9122 24146937, $₫$ e-mail: laskars2000@yahoo.com
Received: 05.06 .2017

Accepted: 22.08 .2017

Published: 30.10 .2017 
PAX3-FKHR fusion gene. Metastatic workup done with whole body positron emission tomography-computed tomography (PET-CT) was negative. The patient was treated with multi-agent chemotherapy as per the IRS-IV protocol [3]. The alveolar histology and suboptimal response after induction CTh mandated the use of radiation therapy for local control. In view of the expected morbidity of EBRT, it was decided to treat this patient using interstitial brachytherapy.

\section{Pre-planning}

Before embarking on the actual implantation procedure, a pre-planning was done to determine ideal catheter positions, safe depth for each catheter, and first/guide catheter position.
With the patient under sedation and the neck in neutral position, the patient was immobilized using a thermoplastic mould and CT images with slice thickness of $1 \mathrm{~mm}$ were obtained on a dedicated CT simulator. Clinical target volume (CTV) was delineated based on the disease extent visible on the planning CT images and modified using the pre CTh MRI. Brachytherapy treatment plans were generated using straight and uniformly spaced catheters placed along the target volume, to get the desired dose distribution. Nasion served as a reference for the first/guide catheter (Figures 2A-C). This guide catheter would then serve as a reference for subsequent catheter positions. Safe penetration depth for each catheter was determined on the axial CT cuts (Figures 2D-F).
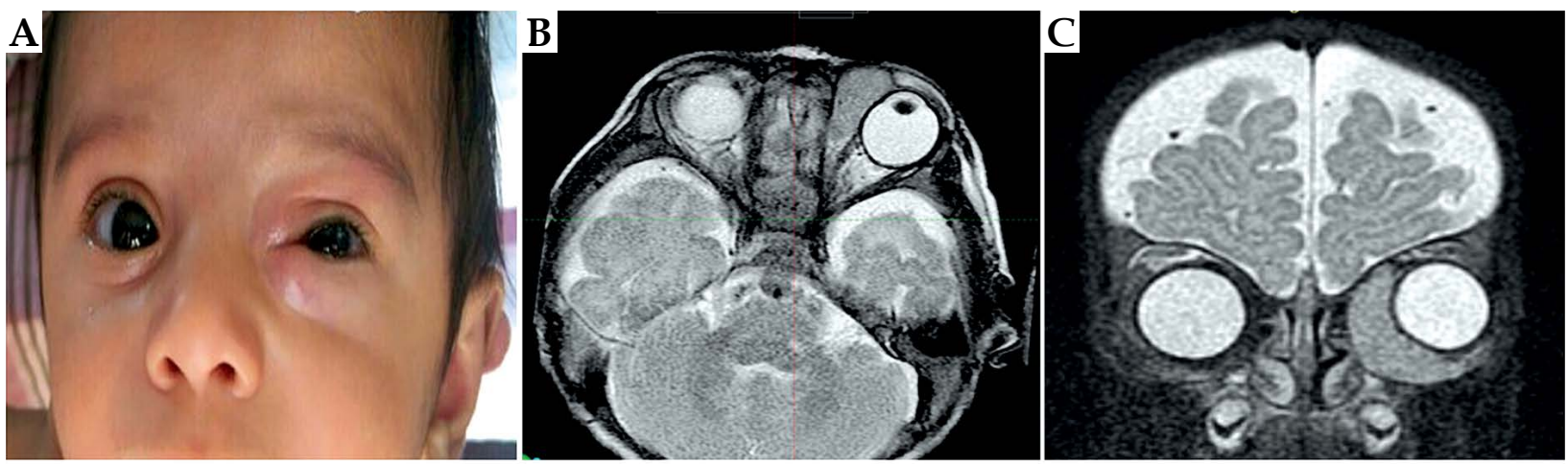

Fig. 1. A) Clinical presentation showing redness and swelling at medial canthus of left eye. B and C) Magnetic resonance imaging of the orbit (T2W axial and coronal) showing homogenous extraconal lesion involving the medial aspect of left orbit
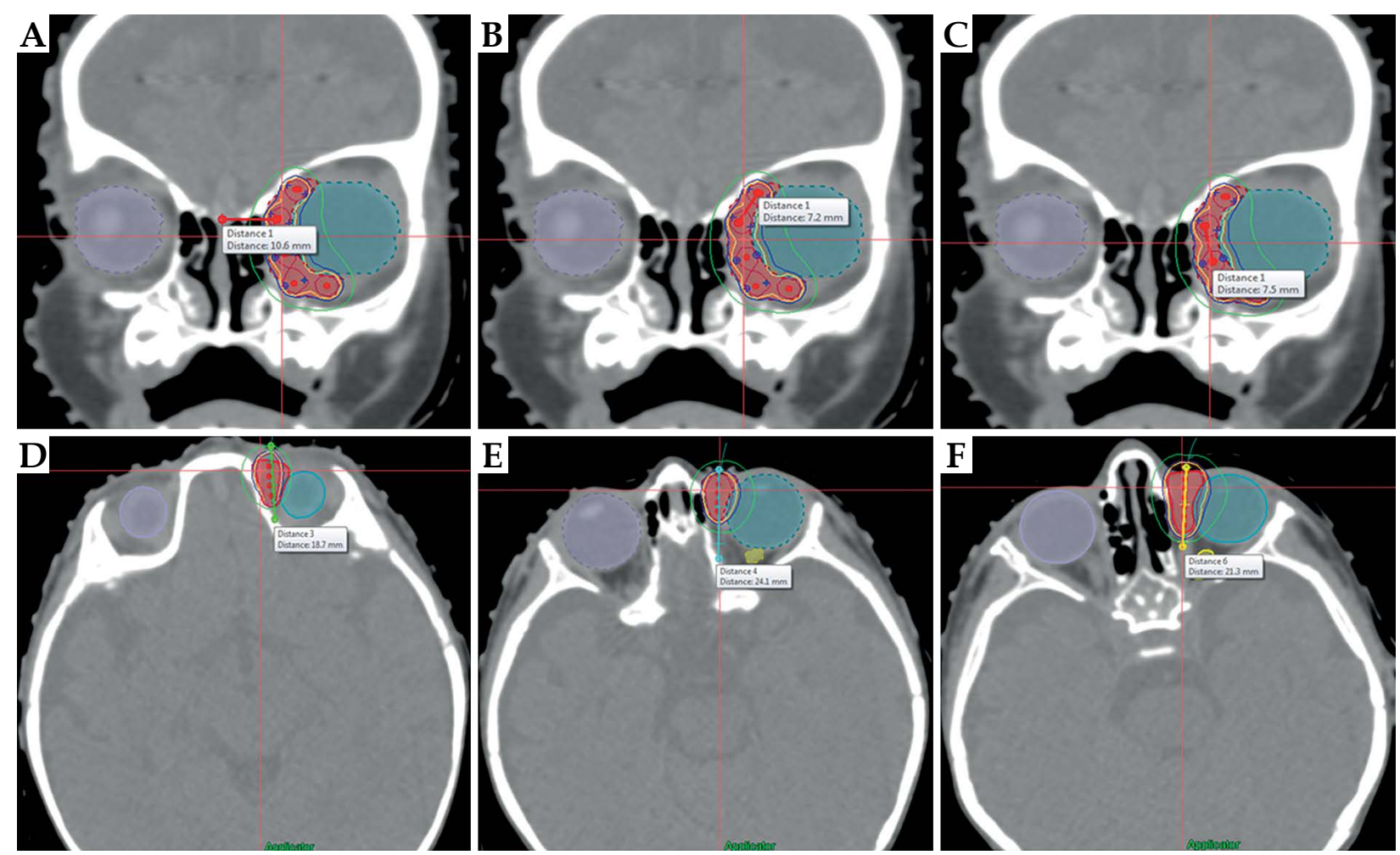

Fig. 2. A) Preplanning images. First/guide catheter entry point determined by measuring the distance from midpoint of the nasion. B and C) All catheter positions determined with reference to the guide catheter. D-F) Measurement of penetration depth for different catheters 

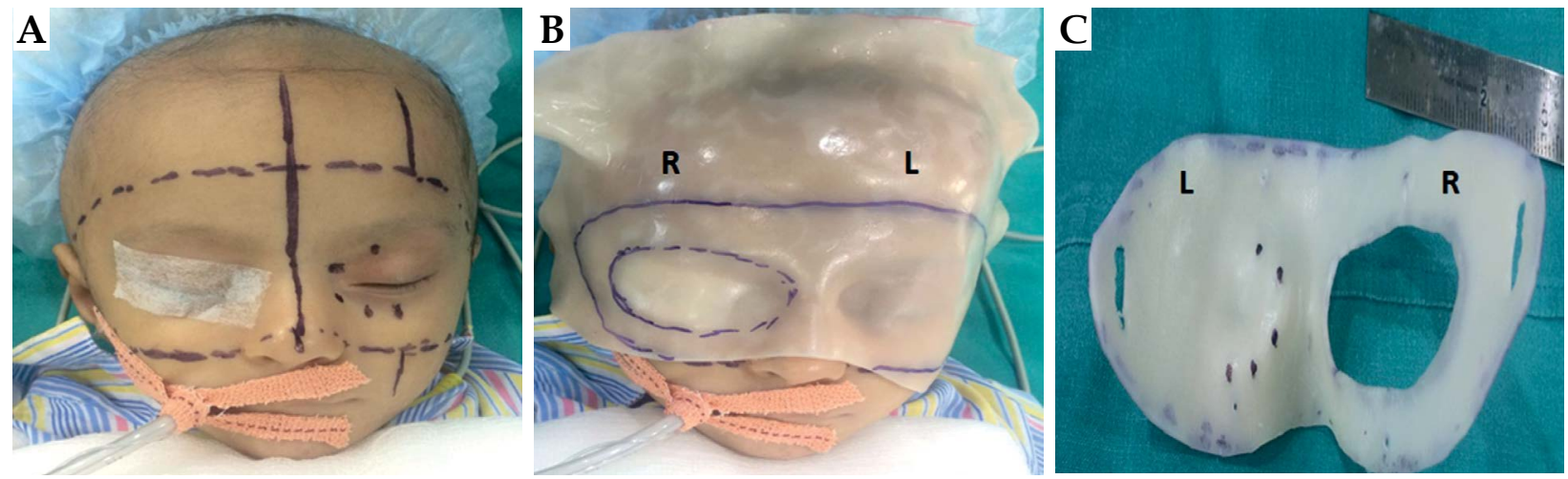

Fig. 3. A) Entry points marked on patient. B) Fabrication of thermoplastic retainer. C) Extrapolation of catheter points onto the retainer

\section{Implantation technique}

With the patient under general anesthesia (G.A.), needle entry points were extrapolated and marked on the skin as determined from the pre-planning CT scan (Figure 3A). The midline, nasion, and bony orbit were used as reference. A customized thermoplastic retainer for the brachytherapy catheters was fashioned in the operating room with the patient in the treatment position (Figure 3B). This retainer would be required to maintain the implant in place through-
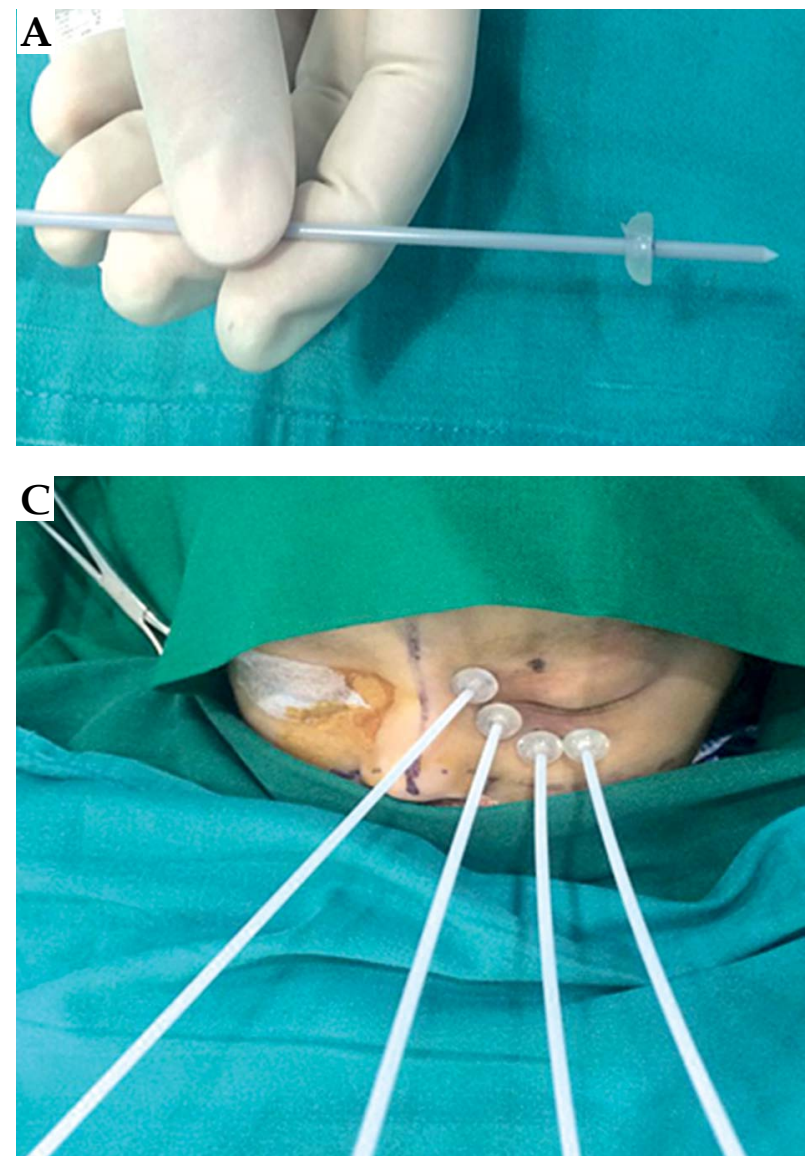

Fig. 4. Implantation technique. A-C) Catheters with washers to guide the depth of insertion. D) Positioning of retainer over the catheters

out the period of delivery, i.e. 4-5 days. The catheter entry points were then transferred onto the retainer (Figure 3C). This retainer would be fastened to the head of the patient using Velcro straps after the procedure.

Using washers to guide the depth of implantation (Figure 4A), determined on the pre-planning CT scan, five brachytherapy catheters (189.601 ProGuide Needle Set 6F, sharp, (Oncentra Brachy, Elekta AB, Stockholm, Sweden) [14]) were implanted carefully, without injuring the eyeball (Figures $4 \mathrm{~A}-\mathrm{C}$ ). Each catheter was then care-
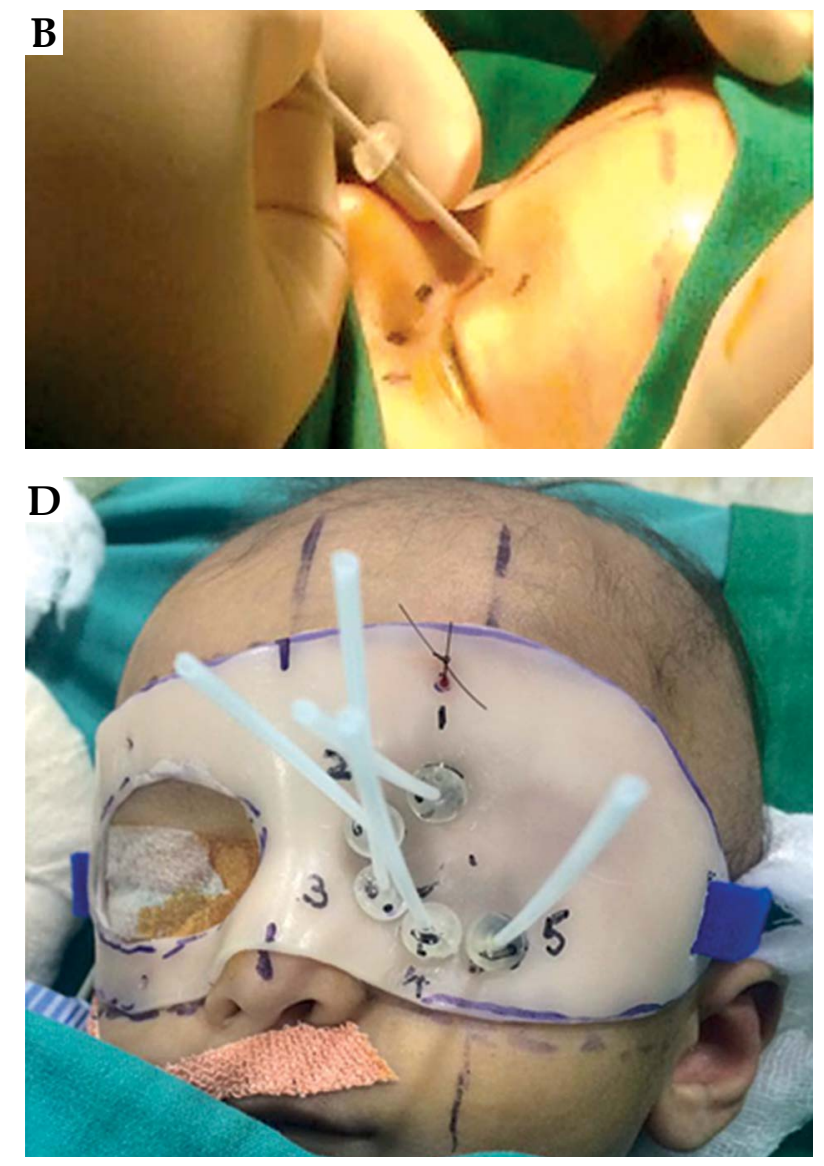

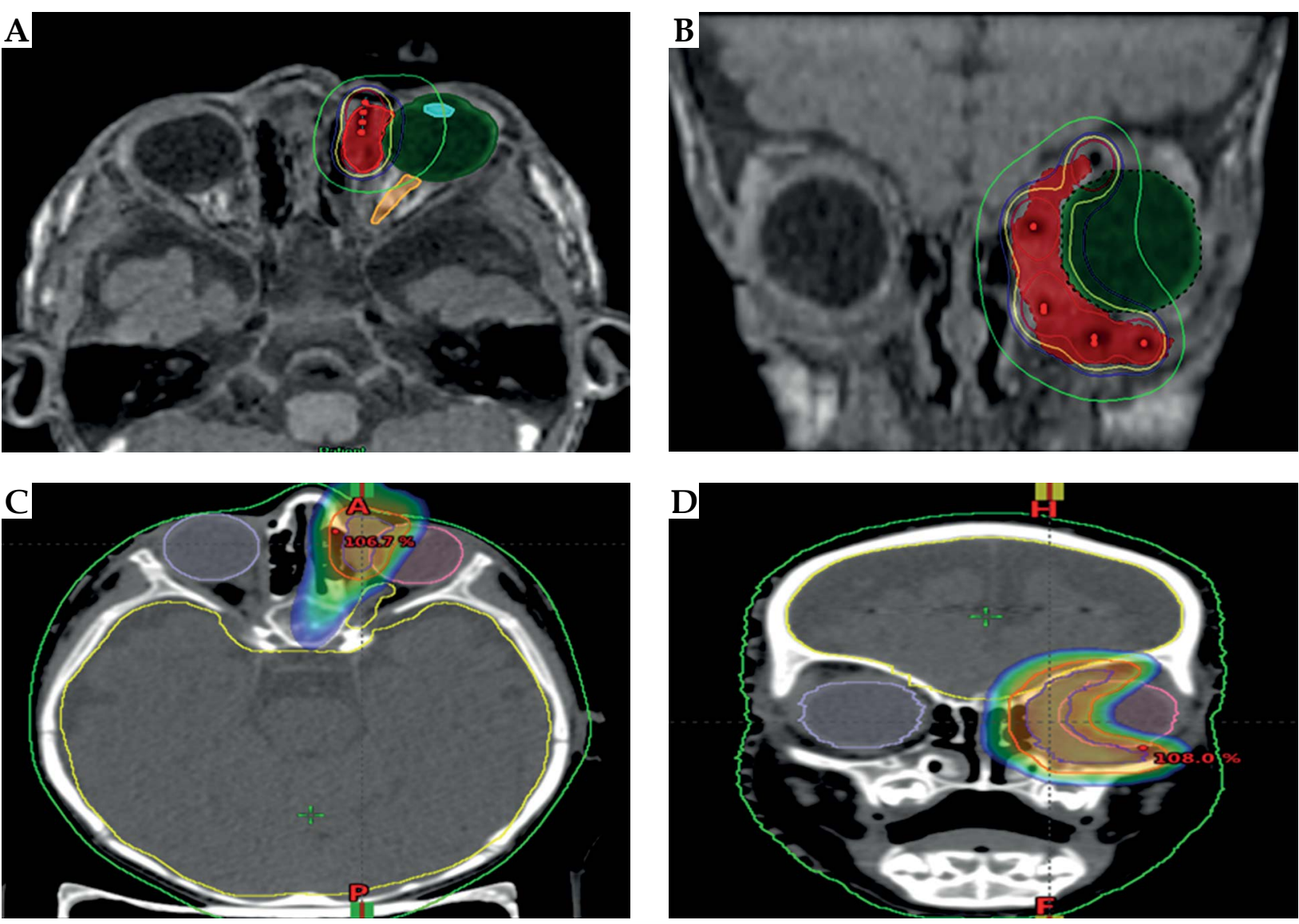

Fig. 5. A and B) High-dose-rate brachytherapy plan on magnetic resonance imaging sequences (isodose lines: yellow - 100\% and green - 50\%). C and D) Rapid arc external beam radiotherapy (EBRT) plan (isodose wash: orange - 95\%, dark blue - 50\%). Note higher doses to pituitary and bony orbit with EBRT

fully passed through the holes drilled into the retainer, which was then guided over the catheters and positioned to fit snugly on the patients face. The catheters were secured using washers and glue. The retainer was secured in place using Velcro straps (Figure 4D).

\section{Results}

\section{Planning and dose prescription}

After the procedure in the operating room, the patient was transferred to MRI suite under G.A. and a planning MRI was done using 3D FSPGR (three-dimensional fast spoiled gradient recall) sequences of $1 \mathrm{~mm}$ slice thickness and $0 \mathrm{~mm}$ gap. Clinical target volume was delineated on the planning images as previously described. ${ }^{192} \mathrm{Ir}$ highdose-rate (HDR) brachytherapy plan was generated using Oncentra brachytherapy planning system (Oncentra Brachy, Elekta AB, Stockholm, Sweden). The dose distribution was evaluated and optimized using graphical optimization to ensure adequate coverage of the target volume by the reference isodose (Figures $5 \mathrm{~A}$ and $5 \mathrm{~B}$ ). The plan was evaluated using dose volume histogram (DVH) for target volume coverage and dose to the critical structures (Table 1). A total dose of 32 Gy was delivered using 4 Gy fractions (two fractions per day) over 4.5 days. For dosimetric comparison with EBRT,
Table 1. Dosimetric parameters of external beam radiotherapy and brachytherapy plans

\begin{tabular}{|c|c|c|}
\hline Structures & $\begin{array}{c}\text { EBRT rapid arc } \\
(\%)\end{array}$ & $\begin{array}{c}\text { Brachytherapy } \\
(\%)\end{array}$ \\
\hline CTV (mean dose) & 101 & 158 \\
\hline$C T V D_{90}$ & 100 & 92 \\
\hline CTV $V_{100}$ & 95 & 90 \\
\hline CTV $V_{150}$ & - & 30 \\
\hline CTV V 200 & - & 16 \\
\hline $\mathrm{DHI}$ & - & 66 \\
\hline Eye left (mean dose) & 61 & 61 \\
\hline Lens left (mean dose) & 66 & 51 \\
\hline Optic nerve left (max dose) & 72 & 54 \\
\hline Bony orbit left (mean dose) & 68 & 39 \\
\hline Pituitary gland (mean dose) & 42 & 6 \\
\hline \multicolumn{3}{|c|}{$\begin{array}{l}\text { EBRT - external beam radiotherapy, } B T \text { - brachytherapy, CTV - clinical targe } \\
\text { volume, } D_{90}-\text { minimum dose received by } 90 \% \text { of the target volume, } V_{100}-\text { vol } \\
\text { ume receiving by } 100 \% \text { of the prescription dose, } V_{150}-\text { volume receiving by } \\
150 \% \text { of the prescription dose, } V_{200}-\text { volume receiving by } 200 \% \text { of the prescrip } \\
\text { tion dose, } D H I \text { (dose homogeneity index) }-\left(V_{100}-V_{150}\right) V_{100}\end{array}$} \\
\hline
\end{tabular}



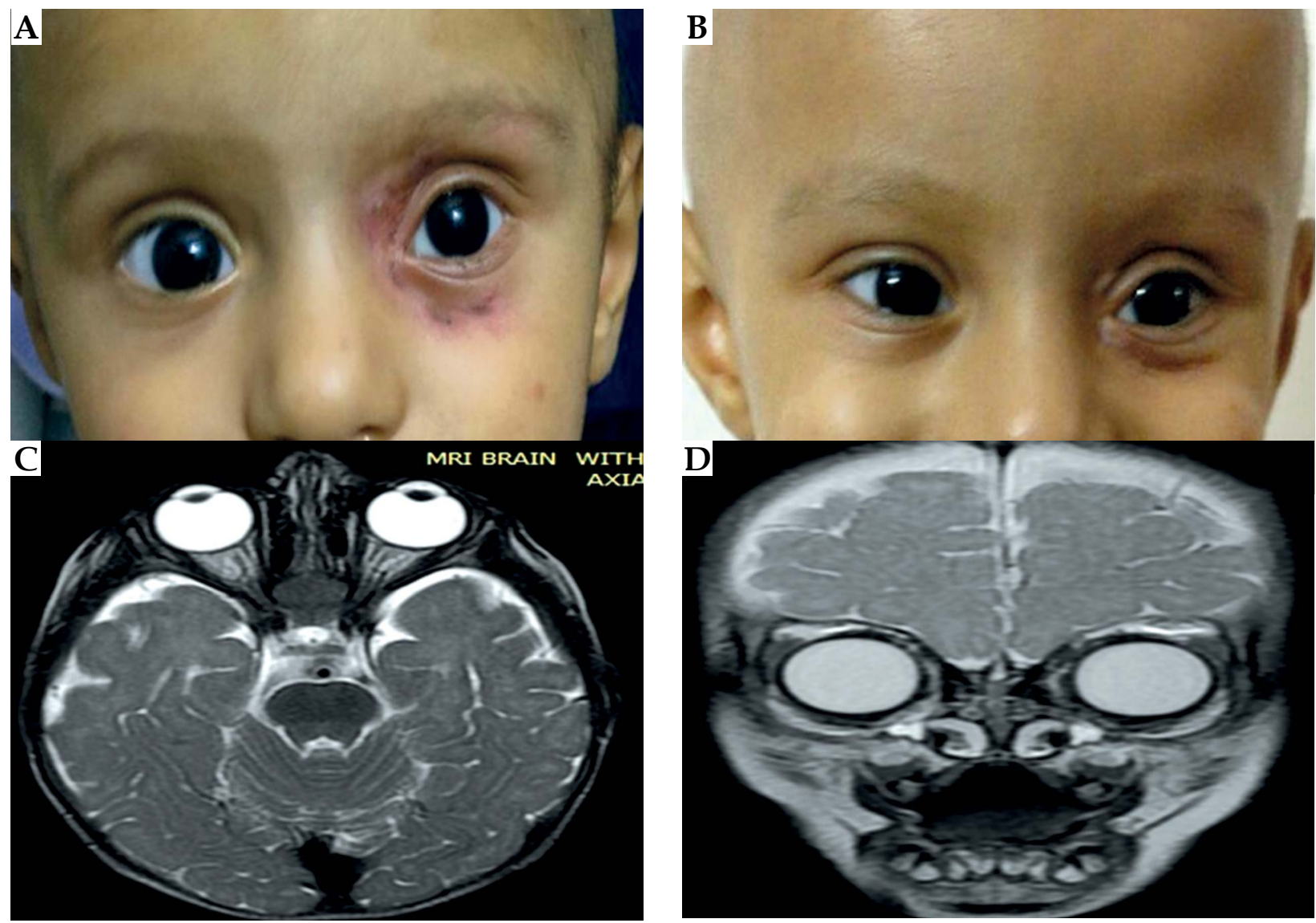

Fig. 6. A and B) Acute and late skin changes, respectively. Note the preservation of eyebrows and excellent cosmetic outcome. $\mathrm{C}$ and D) Post-treatment T2W axial and coronal MRI showing complete regression of tumor

\section{Step 1:}

1. Ideal catheter positions

\section{Pre-planning}

2. Depth of insertion for each catheter

3. Catheter insertion co-ordinates with reference to bony landmarks (Figure 2)

\section{Step 2: \\ 1. Extrapolation of needle entry points on skin \\ 2. Fabrication of customised retainer \\ 3. Transfer entry points onto the retainer and drill holes (Figure 3)}
Step 3:
1. Washers used to guidation technique
2. Needles inserted carefully avoiding injury
3. Retainer guided over catheters secured using wahers and glue
4. Retainer secured using Velcro straps (Figure 4)

\section{Step 4: \\ Treatment planning and delivery \\ 1. MRI based BT planning and optimisation (Figure 5) \\ 2. Plan evaluation using DVH (Table 1) \\ 3. Treatment delivery under sedation}

Fig. 7. Flowchart of the procedure a RapidArc (Eclipse, Varian Medical Systems, Palo Alto, CA) plan was generated using a $3 \mathrm{~mm}$ PTV margin to the CTV (Figures 5C and 5D). The dosimetric comparison between brachytherapy and EBRT are highlighted in Table 1 , and shows dosimetric superiority of brachytherapy for target volume and OAR's.

\section{Treatment delivery and follow-up}

The patient was treated twice daily using sedation, with a minimum 6 hours gap between fractions. Care was taken to ensure avoidance of handling of the retainer by the child. Oral analgesics and antibiotics were continued till the end of treatment. Acute reactions were minimal and consisted of mild skin erythema (Figure 6A).

First follow-up was done at 3 months post brachytherapy. MRI scan of the orbit done at follow-up, showed a complete response with no evidence of disease (Figure 6C and 6D). The patient has completed all treatment and is currently on regular follow-up, and has excellent cosmetic and functional outcome without any restriction of ocular mobility, dryness of the eye, or watering (Figure 6B). Flow-chart of the procedure is represented in Figure 7.

\section{Discussion}

Multiagent chemotherapy and EBRT is the current management standard for orbital RMS. External beam 
radiotherapy has limitations like prolonged treatment of 5-6 weeks, anesthesia for young children, higher doses to unaffected surrounding ocular, bony and intracranial structures, and late toxicities like orbital hypoplasia, facial asymmetry, cataract, dry eye, eye lash loss, and endocrine abnormalities $[4,5,6]$. Even with the best of EBRT techniques like intensity-modulated radiotherapy (IMRT) or proton beam therapy (PBT), a significant dose is received by surrounding orbital structures [15].

Brachytherapy with its rapid dose fall-off beyond the catheters allows the delivery of optimal dose to the target while minimizing the dose to surrounding orbital structures and thus, reducing consequential late toxicities [8,9]. Brachytherapy seems to offer an edge even over PBT in reducing doses to the bony orbit. Indirect comparison with dosimetric data of PBT from Yock et al. [15] shows significantly lower average doses to bony orbit with brachytherapy. This may result in a potentially reduced risk of skeletal deformities and second malignancies. Higher intratumoral doses with brachytherapy results in higher average doses to the target volume and may potentially enhance local control. There are also other advantages, like reduced overall treatment time, reduced requirement of anesthesia, and reduced requirement of strict immobilization.

The implantation technique reported in this article provides a detailed approach to an innovative way of interstitial brachytherapy of extraconal orbital tumors, and unlike other previous reports of orbital brachytherapy, does not require surgical debulking and surgical access for implantation $[8,9,10,11,12,13,16]$. Blank et al. [8] in a group of 20 children with RMS, have described the use of mold brachytherapy intraoperatively following macroscopic resection with minimal late toxicity. Various innovative techniques of interstitial brachytherapy for orbital tumors have also been reported previously $[10,11,13,16]$, with excellent local control and functional outcomes. These reported techniques have been either in combination of function preserving surgery $[11,13,16]$ or after enucleation for melanomas with extra scleral disease extension [10].

With careful case selection and optimum pre-planning, this technique appears to be safe and effective option for radiation therapy even in infants. The relative contraindications of this method would include tumors in close proximity to the optic nerve and tumors adjacent to the orbital apex. Though a long term follow-up would be optimal to assess the effects on visual acuity, ocular motility and orbital development, the early outcomes in terms of disease control, cosmesis, and functional outcome have been encouraging.

\section{Conclusions}

Orbital brachytherapy with its potential benefits of reduced late toxicities and shorter duration of treatment, is an attractive treatment option for very young children with orbital tumors, and should be considered as an alternative to EBRT whenever feasible. Extraconal tumors are best suited for interstitial brachytherapy technique reported in this manuscript. Implementation of this technique as a standard protocol for localized extraconal orbital tumors is being implemented at our institute. We have fur- ther improved our retainer stability and comfort, and also propose to make the procedure safer and more accurately reproducible using ultrasonography guidance for catheter insertion in the future.

\section{Acknowledgements}

POCL Medical Solutions, Mumbai, India, for their help in fabrication of the brachytherapy catheter retainer.

\section{Disclosure}

Authors reports no conflict of interest.

\section{References}

1. Turner JH, Richmon JD. Head and neck rhabdomyosarcoma: a critical analysis of population-based incidence and survival data. Otolaryngol Head Neck Surg 2011; 145: 967-973.

2. Crist W, Gehan EA, Ragab AH et al. The Third Intergroup Rhabdomyosarcoma Study. J Clin Oncol 1995; 13: 610-630.

3. Crist WM, Anderson JR, Meza JL et al. Intergroup rhabdomyosarcoma study-IV: results for patients with nonmetastatic disease. J Clin Oncol 2001; 19: 3091-3102.

4. Finger PT. Radiation therapy for orbital tumors: concepts, current use, and ophthalmic radiation side effects. Surv Ophthalmol 2009; 54: 545-568.

5. Heyn R, Ragab A, Raney RB Jr. et al. Late effects of therapy in orbital rhabdomyosarcoma in children. A report from the Intergroup Rhabdomyosarcoma Study. Cancer 1986; 57: 1738-1743.

6. Paulino AC, Simon JH, Zhen W, Wen BC. Long-term effects in children treated with radiotherapy for head and neck rhabdomyosarcoma. Int J Radiat Oncol Biol Phys 2000; 48: 1489-1495.

7. Rousseau P, Flamant F, Quintana E et al. Primary chemotherapy in rhabdomyosarcomas and other malignant mesenchymal tumors of the orbit: results of the International Society of Pediatric Oncology MMT 84 Study. J Clin Oncol 1994; 12: 516-521.

8. Blank LE, Koedooder K, van der Grient HN et al. Brachytherapy as part of the multidisciplinary treatment of childhood rhabdomyosarcomas of the orbit. Int J Radiat Oncol Biol Phys 2010; 77: 1463-1469.

9. Buwalda J, Schouwenburg PF, Blank LE et al. A novel local treatment strategy for advanced stage head and neck rhabdomyosarcomas in children: results of the AMORE protocol. Eur J Cancer 2003; 39: 1594-1602.

10. Finger PT, Tena LB, Semenova E et al. Extrascleral extension of choroidal melanoma: post-enucleation high-dose-rate interstitial brachytherapy of the orbit. Brachytherapy 2014; 13: 275-280.

11. Kovács G, Rochels R, Mehdorn $H$ et al. Eye preservation brachytherapy for orbital and adjacent tumors: preliminary results. Radiotherapy of Ocular Diseases 1997; 30: 56-64.

12. Lin KL, Tena LB, Finger PT et al. Orbital brachytherapy for extrascleral extension of choroidal melanoma. Br J Ophthalmol 2006; 90: 1438-1439.

13. Meyer JE, Brocks C, Gehrking E et al. Brachytherapy in combination with function-preserving surgery. An interdisciplinary challenge. HNO 2008; 56: 471-478 [In German].

14. Elekta. Brachytherapy Applicator and Accessory Guide.

15. Yock T, Schneider R, Friedmann A et al. Proton radiotherapy for orbital rhabdomyosarcoma: clinical outcome and a dosimetric comparison with photons. Int J Radiat Oncol Biol Phys 2005; 63: 1161-1168.

16. Kovács G. Modern head and neck brachytherapy: from radium towards intensity modulated interventional brachytherapy. J Contemp Brachytherapy 2015; 6: 404-416. 